\title{
Angiotensin II induces microtubule reorganization mediated by a deacetylase SIRT2 in endothelial cells
}

\begin{abstract}
Aiko Hashimoto-Komatsu ${ }^{1}$, Tetsuaki Hirase ${ }^{1,2}$, Machiko Asaka ${ }^{1}$ and Koichi Node ${ }^{1}$
Angiotensin II has been implicated in vascular remodeling. Microtubule composed of tubulins regulates cell shape, migration and survival. Tubulin acetylation has an important role in the control of microtubule structure and microtubule-based cellular functions. In this study, angiotensin II induced disassembly and deacetylation of $\alpha$-tubulin, which were blocked by pretreatment with an angiotensin II type 1 receptor blocker losartan and a sirtuin class deacetylase inhibitor sirtinol, and by depletion of a deacetylase SIRT2 using RNA interference. We investigated the involvement of SIRT2 in angiotensin II-induced endothelial cell migration using the Boyden chamber method. Angiotensin II caused a significant increase in cell migration, which was blocked by pretreatment with sirtinol and SIRT2 depletion. It has been reported that angiotensin II is involved in cytoskeletal reorganization stimulated by mechanical stretch in endothelial cells. We also demonstrated that endothelial cells subjected to a $10 \%$ uniaxial stretch showed vertical alignment to the direction of tension and tubulin deacetylation in the peripheral side of cells, in comparison with control static cells. The mechanical stretch-induced changes of microtubules were blocked by pretreatment with sirtinol and SIRT2 depletion. Immunofluorescence microscopy showed that acetylated tubulin was decreased in platelet-endothelial cell adhesion molecule-1-positive cells in the intima of the aortic walls in mice loaded with angiotensin II, in comparison with mice loaded with control vehicle. These data show that angiotensin II and mechanical stretch stimulate microtubule redistribution and deacetylation via SIRT2 in endothelial cells, suggesting the emerging role of SIRT2 in hypertension-induced vascular remodeling.
\end{abstract}

Hypertension Research (2011) 34, 949-956; doi:10.1038/hr.2011.64; published online 16 June 2011

Keywords: angiotensin II; cyclic stretch; endothelial cells; microtubule; SIRT2

\section{INTRODUCTION}

Angiotensin II is a multifunctional peptide that is the principal effector of the systemic and local renin-angiotensin system. The effects of angiotensin II are mediated by type 1 and 2 plasma membrane receptors that are expressed in vascular cells including endothelial cells, through activation of multiple signaling pathways. A number of studies have shown the involvement of angiotensin II in vascular remodeling, as well as in the regulation of blood pressure and neurohumoral homeostasis. ${ }^{1}$ Mechanical stress against vascular cells also modulates cell function by triggering cell signaling. ${ }^{2}$ Angiotensin II and mechanical stress have important roles in hypertensioninduced vascular remodeling. The cytoskeleton is one of the key targets in cell signaling activated by angiotensin II and mechanical stress in vascular cells.

Microtubules control numerous cellular functions in endothelial cells, including cell shape, mitosis, intracellular transport, adhesion and migration. Endothelial migration is required for vascular remodeling, such as angiogenesis and reendothelialization in wound repair and after angioplasty or bypass procedure. ${ }^{3}$ Microtubules polymerized with heterodimers formed by an $\alpha$ - and a $\beta$-tubulin are one of the major components of the cellular cytoskeletal system. Microtubules show dynamic instability, interconversion of assembly and disassembly. ${ }^{4-6}$ The posttranslational modification of tubulin, such as deacetylation, detyrosination, phosphorylation and polyglutamation, is involved in the regulation of microtubule dynamics and microtubule-based cellular functions such as cell morphology, cell survival and cell migration. Acetylation of $\alpha$-tubulin on lysine- 40 has been implicated in the regulation of microtubule stability and structure, as well as microtubule-based cellular functions, suggesting that acetylation is one of the most important modifications of tubulin.,

Silent information regulator 2 was identified in Saccharomyces cerevisiae as a nicotinamide adenine dinucleotide ${ }^{+}$-dependent histone deacetylase. Seven homologs of silent information regulator 2 have been identified in mammals and designated as SIRT1-7. The SIRT proteins show distinct tissue and cellular distribution that suggests diverse functions of each SIRT protein. ${ }^{9}$ Human SIRT2 is colocalized with microtubules in the cytoplasm and deacetylates lysine- 40 of $\alpha$ tubulin both in vitro and in vivo. SIRT2 depletion by RNA interference causes tubulin hyperacetylation. ${ }^{10}$ Therefore, SIRT2 is considered to be a tubulin deacetylase. SIRT2 is involved in cell division and

\footnotetext{
${ }^{1}$ Department of Cardiovascular Medicine, Saga University Faculty of Medicine, Saga, Japan and ${ }^{2}$ Department of Bioscience and Genetics, National Cerebral and Cardiovascular Center Research Institute, Suita, Japan

Correspondence: Dr T Hirase, Department of Bioscience and Genetics, National Cerebral and Cardiovascular Center Research Institute, 5-7-1 Fujishiro-dai, Suita 565-8565, Japan. 
myelinogenesis, whereas the roles of SIRT2 in vascular cells are not fully understood.

In this study, we investigated the effect of angiotensin II on microtubule dynamics, especially on posttranslational modification of tubulin in cultured human endothelial EA hy.926 cells. ${ }^{11}$ Angiotensin II-induced tubulin deacetylation was blocked by pharmacological and genetic inhibition of SIRT2, a tubulin deacetylase. The roles of microtubule and tubulin acetylation in endothelial migration induced by angiotensin II were investigated using the Boyden chamber method. In addition, mechanical stretch caused microtubule reorganization and tubulin deacetylation mediated by angiotensin II type 1 receptor and SIRT2 in endothelial cells. Tubulin acetylation was decreased in aortic endothelial cells loaded with angiotensin II in mice in vivo. Therefore, this study shows that angiotensin II and mechanical stretch induce SIRT2-mediated microtubule reorganization in endothelial cells, suggesting the emerging role of SIRT2 in hypertension-induced vascular remodeling.

\section{METHODS}

\section{Materials}

Angiotensin II and PD123319 were purchased from Sigma (St Louis, MO, USA). Sirtinol was purchased from CALBIOCHEM (La Jolla, CA, USA). Anti-acetylated tubulin (6-11 B-1) antibody was purchased from Sigma. Anti- $\alpha$-tubulin (ab15246) antibody was purchased from Abcam (Cambridge, MA, USA). Anti-SIRT2 antibody was purchased from Santa Cruz Biotechnology (Santa Cruz, CA, USA). FITC-conjugated and Alexa 488-conjugated antimouse antibody and Alexa 568-conjugated anti-rabbit antibody were obtained from Molecular Probes (Eugene, OR, USA).

\section{Cell culture}

EA hy.926 cells, a gift from Dr Cora-Jean S Edgell (University of North Carolina at Chapel Hill, Chapel Hill, NC, USA), were maintained at $37^{\circ} \mathrm{C}$ in an atmosphere of $5 \% \mathrm{CO}_{2}$ in Dulbecco's modied Eagle's medium containing $5 \%$ fetal bovine serum, $100 \mathrm{U} \mathrm{ml}^{-1}$ penicillin and $100 \mu \mathrm{g} \mathrm{ml}^{-1}$ streptomycin. ${ }^{11}$

\section{Gel electrophoresis and immunoblotting}

The protein contents of the samples were determined using a Bio-Rad protein assay (Bio-Rad Laboratories, Hercules, CA, USA). The samples ( $40 \mu \mathrm{g}$ protein) were resolved by one-dimensional sodium dodecyl sulfate-polyacrylamide gel electrophoresis and then electrophoretically transferred to polyvinylidene difluoride membranes (ATTO, Tokyo, Japan). The membranes were subjected to immunoblotting as described previously. ${ }^{12}$

\section{Immunofluorescence and confocal microscopy}

Cells cultured on coverslips were fixed with 3\% paraformaldehyde for $15 \mathrm{~min}$ and then rinsed with phosphate buffered saline. The cells were permeabilized for $15 \mathrm{~min}$ with phosphate buffered saline containing $0.5 \%$ Triton-X. The cells were blocked with phosphate buffered saline containing $1 \%$ bovine serum albumin for $30 \mathrm{~min}$ and incubated with an anti- $\alpha$-tubulin antibody and an anti-acetylated tubulin antibody for $1 \mathrm{~h}$. After rinsing, cells were incubated for 30 min with secondary antibodies conjugated with Alexa 488 or Alexa 546 (Molecular Probes), rinsed and mounted. For nuclear staining, TO-PRO3 from Invitrogen (Carlsbad, CA, USA) was used. Images were captured using confocal microscopy (LSM5 Pascal Ver 3.2, Carl Zwiss, Jena, Germany), at a magnification of 630. Levels of $\alpha$-tubulin and acetylated tubulin were evaluated by measuring the fluorescence intensity of confocal microscopy images from 10 samples in three independent experiments using the ImageJ software program (NIH, Bethesda, MD, USA).

\section{Transfection of small interfering RNA (siRNA)}

Silencer pre-designed siRNA SIRT2 (Ambion Accession No. AM16708, ID; 19657, purchased from Ambion, Austin, TX, USA) was used following the manufacturer's protocol. Transfection of siRNA was performed at a concentration of $100 \mathrm{nmoll}^{-1}$ using lipofectamin RNAiMAX (Invitrogen).

\section{Migration assay (the Boyden chamber method)}

The lower chamber of a Boyden chamber was filled with media containing $100 \mathrm{nmoll}^{-1}$ angiotensin II or control vehicle. A membrane filter pre-coated with $0.25 \%$ gelatin was placed between the chambers. Endothelial cells were trypsinized and suspended in Dulbecco's modied Eagle's medium and counted. A total of $2 \times 10^{3}$ cells were re-suspended and placed in the upper chamber of a Boyden chamber. After $2 \mathrm{~h}$ of incubation at $37^{\circ} \mathrm{C}$, the cells on the upper side of the membrane were gently scraped off. Then cells were fixed with the fixative for $5 \mathrm{~s}$ and stained with Diff-Quick Solution 1 and 2 (Sysmex, Kobe, Japan). Transmigrated cells of three chambers were counted in high-power $(\times 100)$ microscope fields in three independent experiments.

\section{Cyclic stretch of cells}

EA hy.926 cells were cultured in silicone chamber coated with human fibronectin. After overnight incubation at $37^{\circ} \mathrm{C}$, the chambers were applied a uniaxial sinusoidal stretch of $10 \%$ at a frequency of 50 cycles $\min ^{-1}(0.83 \mathrm{~Hz})$ for $2 \mathrm{~h}$ using model ST-140 (STREX, Osaka, Japan).

\section{Animals}

Male mice aged 32 weeks (C57BL/6J strain, CLEA Japan, Tokyo, Japan) with an average weight of $29-35 \mathrm{~g}$ were used in this study. The mice were implanted s.c. with an osmotic mini-pump (Alzet 2004; DURECT Co., Cupertino, CA, USA) containing saline alone or angiotensin II (Sigma Chemical, Palo Alto, CA, USA) dissolved in saline that was delivered at the rate of $1.44 \mathrm{mg} \mathrm{kg}^{-1} \mathrm{~d}^{-1}$ for 4 weeks ( $n=3$ per group). After the mice were anesthetized with pentobarbital sodium ( $80 \mathrm{mg} \mathrm{kg}^{-1}$ i.p.; Abbot Laboratories, Abbott Park, IL, USA), the heart was perfused with saline containing $10 \mathrm{U} \mathrm{ml}^{-1}$ heparin. The aortic sample was dissected and fixed in $4 \%$ paraformaldehyde overnight and then embedded in Tissue-Tek OCT compound (Sakura Finetek, Tokyo, Japan). All experiments were approved by the institutional animal research committee of Saga University and conformed to the animal care guidelines of the American Physiological Society. Five non-consecutive sections chosen from each mouse were stained by immunofluorescence to visualize $\alpha$-tubulin and acetylated tubulin. The fluorescence intensity of images obtained using Axiovision (Carl Zeiss) was analyzed by the ImageJ software program.

\section{Statistical analysis}

Data were expressed as the mean \pm s.e.m. based on three independent experiments. Statistical analysis was performed by Turkey-Kramer post hoc test. Values of $P<0.05$ were considered to be statistically significant.

\section{RESULTS}

\section{Angiotensin II-induced microtubule reorganization and} tubulin deacetylation

$\alpha$-Tubulin was visualized by immunofluorescence in EA hy.926 cells stimulated with angiotensin II or control vehicle to investigate the effects of angiotensin II on microtubule reorganization in endothelial cells (Figure 1A). In control cells, $\alpha$-tubulin was distributed as a radial layout in cell bodies and acetylated tubulin was observed (Figure 1A, a-d). Angiotensin II-treated cells showed the formation of an $\alpha$-tubulin ring formation in the peripheral edge and acetylated tubulin was decreased in comparison with control cells (Figure 1A, e-h, i-l). Similar changes were observed in the cells treated with thrombin, which has been shown to be an inducer of $\alpha$-tubulin redistribution and tubulin deacetylation (Figure $1 \mathrm{~A}, \mathrm{~m}-\mathrm{p}$ ). Whole cell lysates extracted from cells treated with control vehicle, angiotensin II and thrombin were analyzed by immunoblotting (Figure 1B). The protein levels of $\alpha$-tubulin were similar in any type of cells, whereas acetylated tubulin levels were decreased by treatment with angiotensin II and thrombin. The acetylated tubulin levels adjusted with $\alpha$-tubulin levels showed a significant dose-dependent decrease in response to angiotensin II. In addition, angiotensin II treatment decreased the acetylated tubulin levels adjusted with $\alpha$-tubulin levels in a time-dependent manner in EA hy.926 cells (Figure 1C). These data suggest that 
angiotensin II causes $\alpha$-tubulin redistribution and tubulin deacetylation in endothelial cells.

The effects of angiotensin II are transduced by cell surface G protein-coupled receptors named type 1 and type 2 receptors. Pharmacological antagonists against angiotensin II type 1 and type 2 receptors were used to study the involvement of these receptors in angiotensin II-induced $\alpha$-tubulin redistribution and tubulin deacetylation (Figure 2A). Immunofluorescence microscopy showed

A
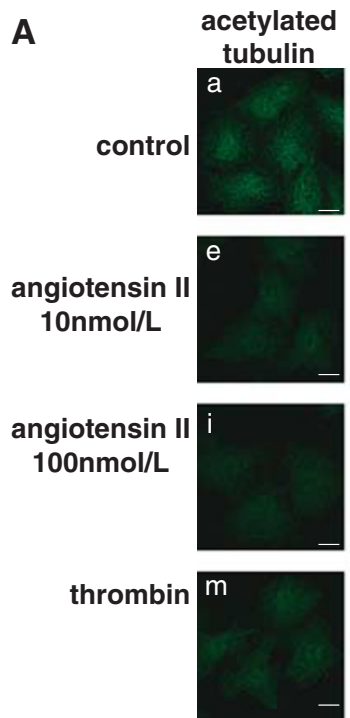

thrombin $\mathrm{m}$
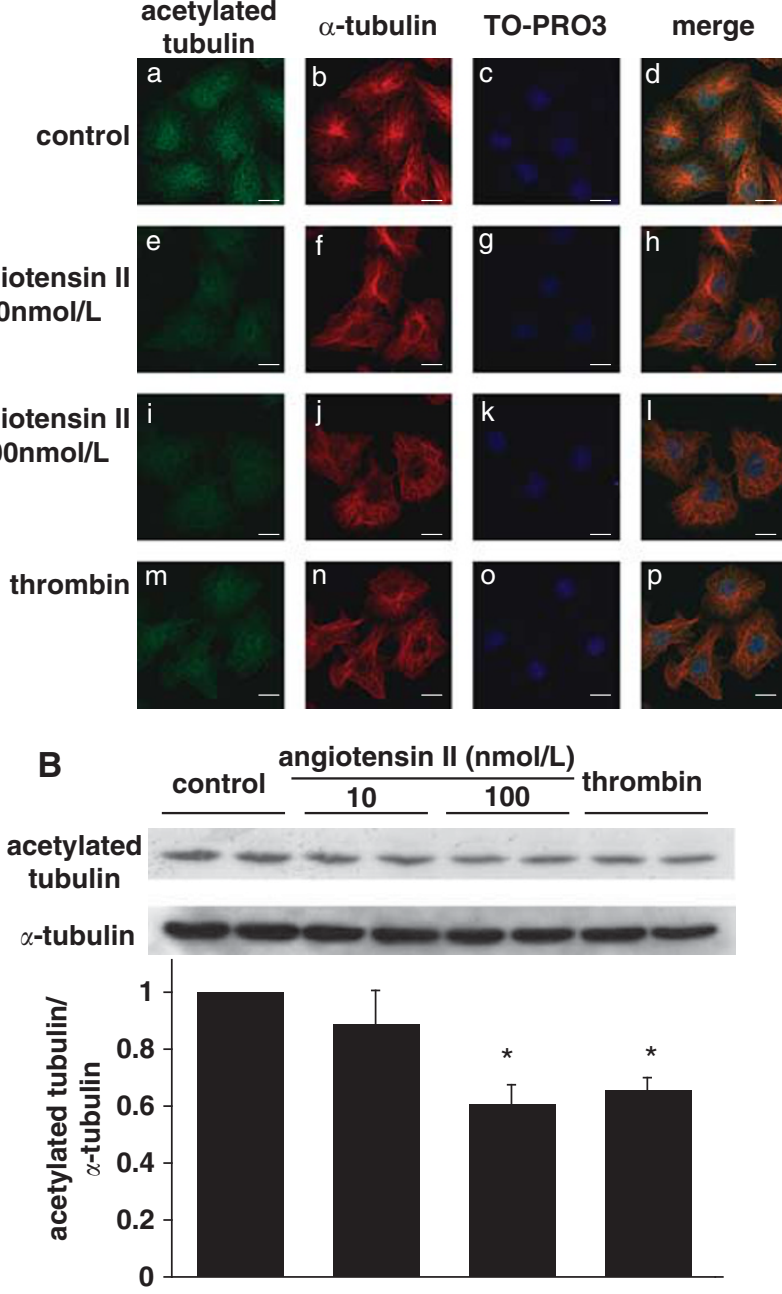

$$
\text { C control angiotenin II (min) } \frac{\text { and }}{5} \text { thrombin }
$$

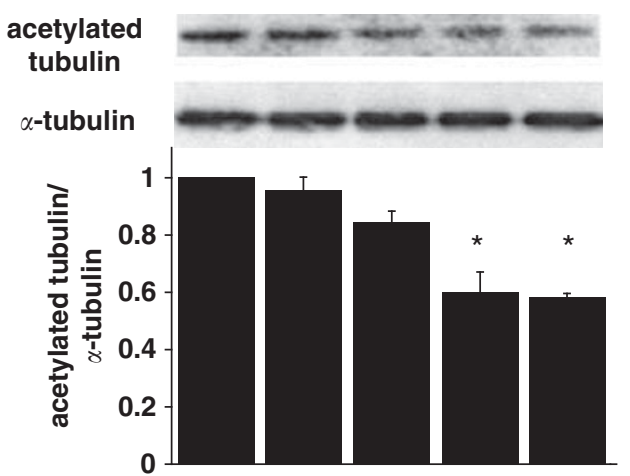

that $\alpha$-tubulin redistribution and tubulin deacetylation induced by angiotensin II were partially blocked in the presence of losartan, an angiotensin II type 1 receptor antagonist, but not PD123319, an angiotensin II type 2 receptor antagonist (Figure 2A, g-l). The protein levels of acetylated tubulin in whole cell lysates were examined by immunoblotting (Figure 2B). Protein levels of $\alpha$-tubulin were similar in all types of cells, whereas angiotensin II-induced decrease of acetylated tubulin was partially blocked in the presence of losartan but not PD123319. The acetylated tubulin levels adjusted with $\alpha$-tubulin levels showed no significant difference between cells treated with angiotensin II and cells treated with angiotensin II in the presence of PD123319, but not losartan. These data indicate the involvement of angiotensin II type 1 receptor in $\alpha$-tubulin redistribution and tubulin deacetylation induced by angiotensin II in endothelial cells.

SIRT2 mediates $\alpha$-tubulin redistribution and tubulin deacetylation induced by angiotensin II

The effects of a specific sirtuin 2 inhibitor, sirtinol (2-[(2-hydroxynaphthalen-1-ylmethylene)amino]- $N$-(1-phenethyl)benzamide), were examined in EA hy.926 cells to investigate the involvement of SIRT2 in angiotensin II-induced $\alpha$-tubulin redistribution and tubulin deacetylation (Figure 3A). Confocal immunofluorescence microscopy revealed that treatment with sirtinol had no significant effect on the distribution of $\alpha$-tubulin and in the levels of acetylated tubulin (Figure $3 \mathrm{~A}, \mathrm{j}-\mathrm{l}$ ). Sirtinol prevented $\alpha$-tubulin redistribution and tubulin deacetylation induced by angiotensin II (Figure $3 \mathrm{~A}, \mathrm{~d}-\mathrm{i}$ ). Immunoblots using whole-cell lysates showed that the decrease in the acetylated tubulin levels by angiotensin II was significantly inhibited in the presence of sirtinol (Figure 3B).

The effects of SIRT2 silencing using siRNA on angiotensin IIinduced decrease of acetylated tubulin were also examined. Transfection of cells with siRNA against SIRT2 dose-dependently reduced the expression of SIRT2 protein in EA hy.926 cells in comparison with control siRNA (Figure 4A). Transfection of cells with siRNA against SIRT2 and control siRNA showed no obvious effects on acetylated tubulin levels in cells treated with control vehicle (Figure 4B). In contrast, transfection of cells with siRNA against SIRT2 inhibited angiotensin II-induced decrease of acetylated tubulin. These data show that SIRT2 mediates $\alpha$-tubulin redistribution and tubulin deacetylation induced by angiotensin II in endothelial cells.

Figure 1 (A) EA hy.926 cells treated with control vehicle (a-d), angiotensin II (10 nmol I-1 (e-h); $\left.100 \mathrm{nmoll}^{-1}(\mathbf{i}-\mathbf{l})\right)$ or thrombin $\left(2 \mathrm{U} \mathrm{ml}^{-1}\right)(\mathbf{m}-\mathbf{p})$ for $60 \mathrm{~min}$ were stained with anti-acetylated tubulin antibody (green), anti$\alpha$-tubulin antibody (red) and TO-PRO3 for nuclear staining (blue) and examined by confocal microscopy. Bar: $20 \mu \mathrm{m}$. (B) Whole cell lysates prepared from EA hy.926 cells that were treated with control vehicle, angiotensin II $\left(10 \mathrm{nmoll}^{-1}, 100 \mathrm{nmoll}^{-1}\right)$ or thrombin $\left(2 \mathrm{U} \mathrm{ml}^{-1}\right)$ for $60 \mathrm{~min}$ were resolved by sodium dodecyl sulfate-polyacrylamide gel electrophoresis and analyzed by immunoblotting with anti-acetylated tubulin antibody and anti- $\alpha$-tubulin antibody. Band intensity measured by scanning immunoblots was analyzed by NIH Image software and adjusted with that of $\alpha$-tubulin. Data shown are the fold increase in comparison with the value for control of three independent experiments. ${ }^{*} P<0.05 \mathrm{vs}$. control. (C) Whole cell lysates extracted from EA hy.926 cells that were treated with control vehicle, angiotensin $\mathrm{II}\left(100 \mathrm{nmolI}^{-1}\right)$ or thrombin for $0,5,15,30$ or $60 \mathrm{~min}$ were analyzed by immunoblotting. Band intensity measured by scanning immunoblots was analyzed by NIH Image software and adjusted with that of $\alpha$-tubulin. The data shown are the fold increase in comparison with the value for control of three independent experiments. ${ }^{*} P<0.05 \mathrm{vs}$. control. 
A

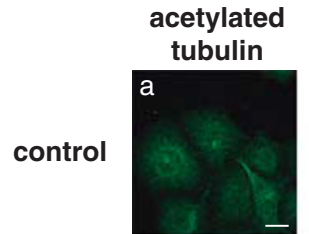

$\alpha$-tubulin
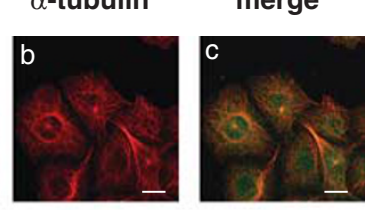

angiotensin II
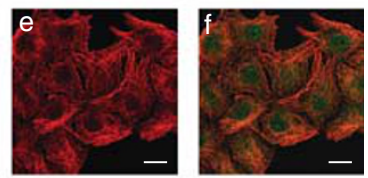

angiotensin II$$
+
$$

losartan
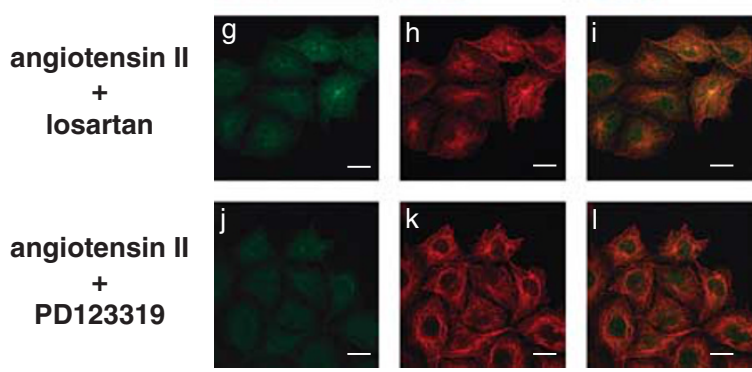

B
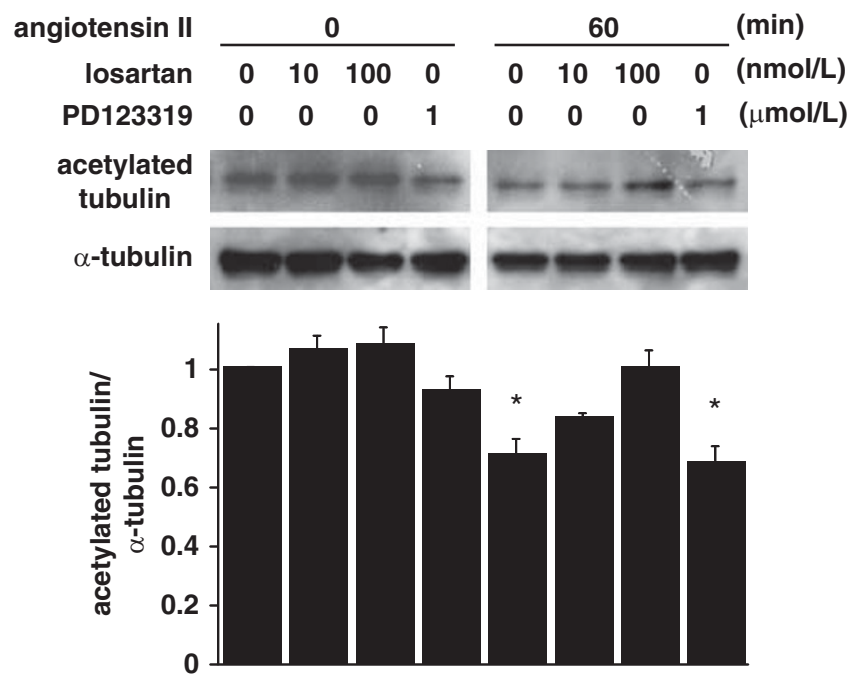

Figure 2 (A) EA hy.926 cells stimulated with vehicle (a-c) or angiotensin II (100 nmol I-1, $60 \mathrm{~min})$ after pretreatment with control vehicle (d-f), losartan $\left(100 \mathrm{nmol} \mathrm{I}^{-1}\right)(\mathrm{g}-\mathrm{i})$ or PD123319 $\left(1 \mu \mathrm{moll}^{-1}\right)(\mathbf{j}-\mathrm{I})$ for $30 \mathrm{~min}$ were stained with anti-acetylated tubulin antibody (green) and anti- $\alpha$-tubulin antibody (red) and then examined by confocal microscopy. Bar: $20 \mu \mathrm{m}$. (B) Whole cell lysates extracted from EA hy.926 cells stimulated with vehicle or angiotensin II (100 nmol I-1, $60 \mathrm{~min})$ after pretreatment with control vehicle, losartan $\left(100 \mathrm{nmol} \mathrm{I}^{-1}\right)$ or PD123319 $\left(1 \mu \mathrm{mol} \mathrm{I}^{-1}\right)$ for $30 \mathrm{~min}$ were analyzed by immunoblotting. The band intensity measured by scanning immunoblots was analyzed by NIH Image software and adjusted with that of $\alpha$-tubulin. The data shown are the fold increase in comparison with the value for control of three independent experiments. ${ }^{*} P<0.05 \mathrm{vs}$. control.

\section{Involvement of SIRT2 in angiotensin II-induced endothelial migration}

The reorganization of microtubules induced by chemotactic factors has been implicated in cell migration. Cell migration was evaluated using the Boyden chamber method, in which cells in the upper chamber migrate across a filter to the lower chamber in response to a gradient of chemoattractants. As shown in Figure 5A, angiotensin II
A
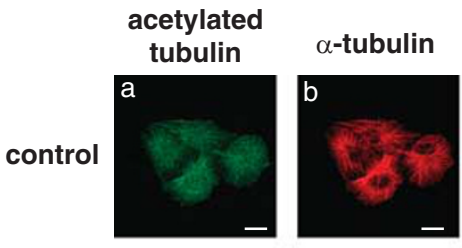

merge
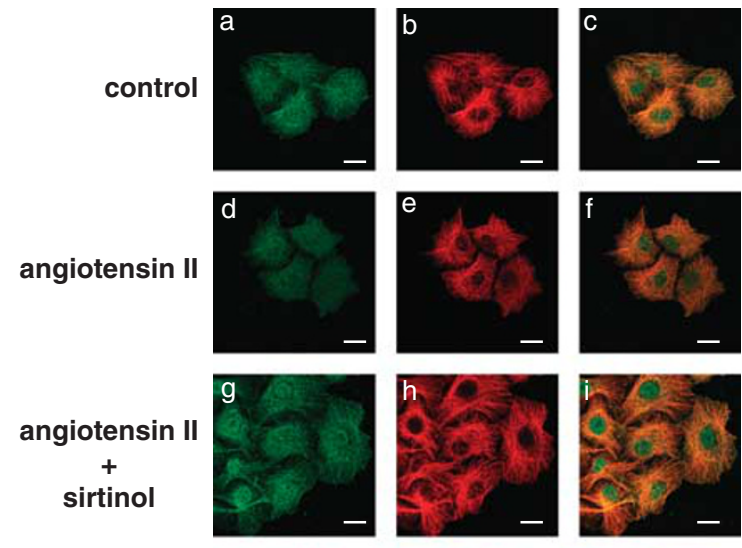

sirtinol
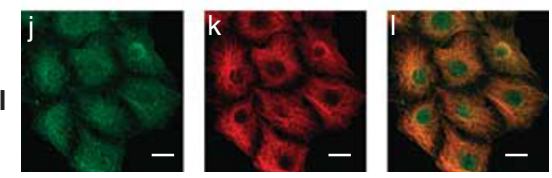

B
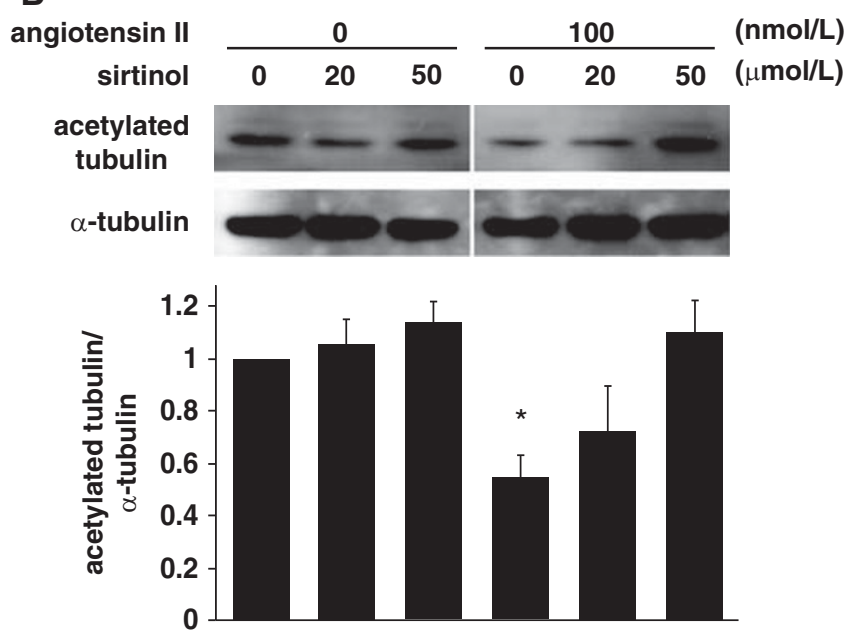

Figure 3 (A) EA hy.926 cells stimulated with vehicle $(\mathbf{a}-\mathbf{c}, \mathbf{j}-\mathbf{I})$ or angiotensin II (100 $\left.\mathrm{nmol} \mathrm{I}^{-1}, 60 \mathrm{~min}\right)$ after pretreatment with vehicle (d-f) or sirtinol (2-[(2-hydroxynaphthalen-1-ylmethylene)amino]- $\mathrm{N}$-(1-phenethyl) benzamide) $\left(20 \mu \mathrm{mol} \mathrm{I}^{-1}, 24 \mathrm{~h}\right)(\mathrm{g}-\mathrm{I})$ were stained with anti-acetylated tubulin antibody (green) and anti- $\alpha$-tubulin antibody (red) and then examined by confocal microscopy. Bar: $20 \mu \mathrm{m}$. (B) Whole cell lysates extracted from EA hy.926 cells stimulated with vehicle or angiotensin II after pretreatment with vehicle or sirtinol $\left(20 \mu \mathrm{mol} \mathrm{I}^{-1}, 24 \mathrm{~h}\right)$ were analyzed by immunoblotting. The band intensity measured by scanning immunoblots was analyzed by the NIH Image software and adjusted with that of $\alpha$ tubulin. The data shown are the fold increase in comparison with the value for control of three independent experiments. ${ }^{*} P<0.05$ vs. control.

stimulated cell migration in EA hy.926 cells. Co-incubation with losartan, but not with PD123319, inhibited angiotensin II-stimulated cell migration. Co-incubation with sirtinol also prevented angiotensin II-induced cell migration. The transfection of cells with siRNA against SIRT2 inhibited angiotensin II-stimulated cell migration, in contrast to control siRNA transfection (Figure 5B). These data suggest that SIRT2 is involved in angiotensin II-stimulated endothelial cell migration. 

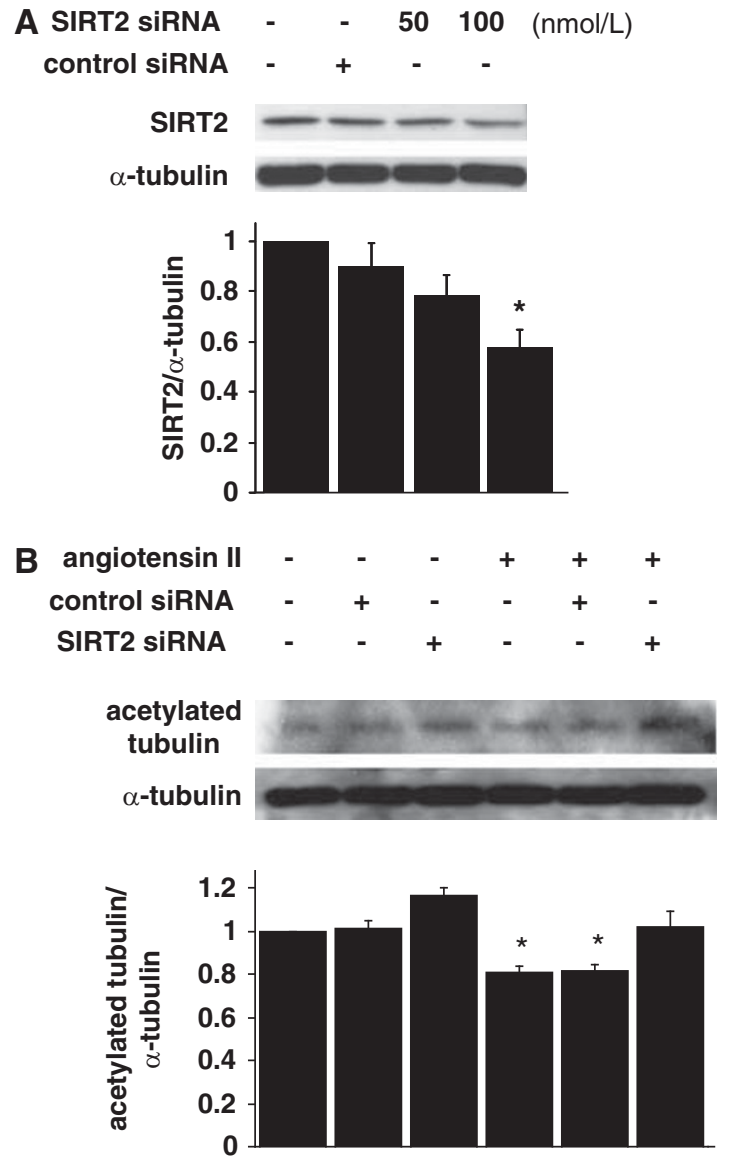

Figure 4 (A) Whole cell lysates from EA hy.926 cells transfected with control siRNA and siRNA against SIRT2 were analyzed by immunoblotting. Band intensity measured by scanning immunoblots was analyzed by $\mathrm{NIH}$ Image software and adjusted with that of $\alpha$-tubulin. The data shown are the fold increase in comparison with the value for control of three independent experiments. ${ }^{*} P<0.05$ vs. control. (B) EA hy.926 cells transfected with control siNRA or siRNA against SIRT2 were stimulated with angiotensin II $\left(100 \mathrm{nmolI}^{-1}, 60 \mathrm{~min}\right)$ or vehicle. Whole cell lysates were analyzed by immunoblotting. Band intensity measured by scanning immunoblots was analyzed by the NIH Image software and adjusted with that of $\alpha$-tubulin. The data shown are the fold increase in comparison with the value for control of three independent experiments. ${ }^{*} P<0.05$ vs. control.

Mechanical stretch-induced microtubule reorganization and $\alpha$-tubulin deacetylation are mediated by angiotensin II type 1 receptor and SIRT2

Angiotensin II has been implicated in cytoskeletal reorganization stimulated by mechanical stretch in endothelial cells that participates in vascular remodeling. EA hy.926 cells cultured on silicone dishes were subjected to $10 \%$ uniaxial cyclic stretch for $2 \mathrm{~h}$ and examined by immunofluorescence to investigate the effects of mechanical stretch on microtubule reorganization. As shown in Figure 6, mechanically stretched EA hy.926 cells showed vertical alignment to the direction of tension and $\alpha$-tubulin distributed in a ring formation accompanied by tubulin deacetylation on the peripheral edge of cells, in contrast to mechanically unloaded control cells that showed $\alpha$-tubulin distribution as a radial pattern in cell bodies. Cellular acetylated tubulin levels adjusted with $\alpha$-tubulin levels were decreased by mechanical stretch (Figure 6). Mechanical stretch-induced changes of microtubules were blocked by pretreatment with losartan and sirtinol (Figure 6). SIRT2 depletion using
A

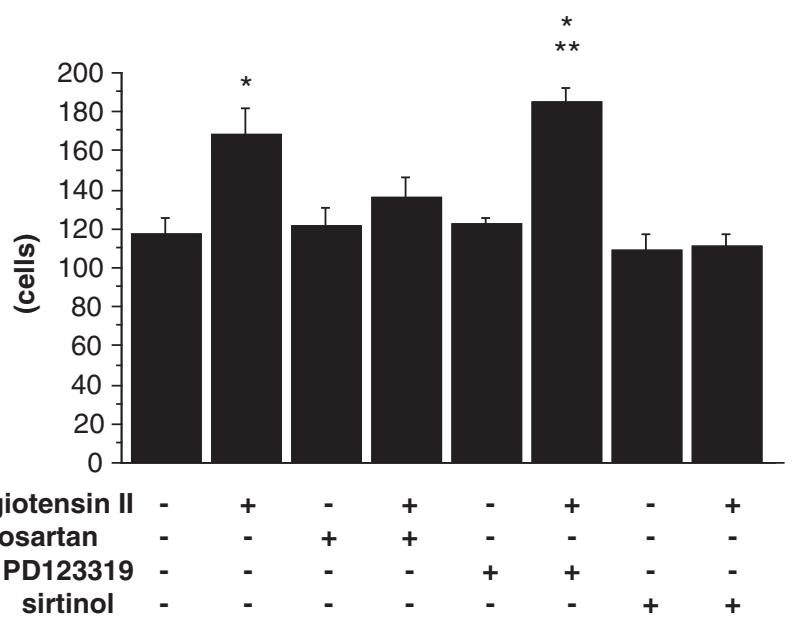

B

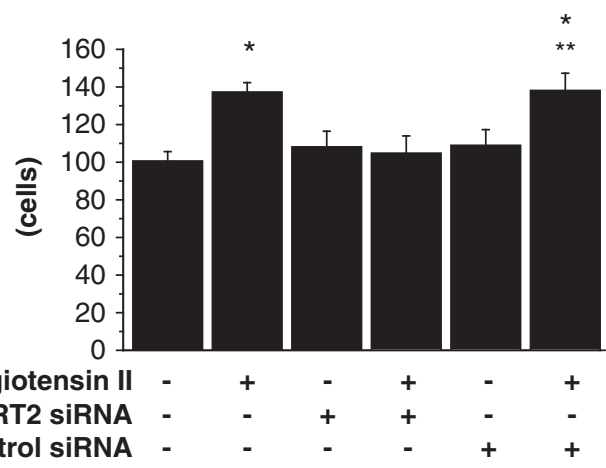

Figure 5 Migration of EA hy.926 cells was evaluated by the Boyden chamber method, as described in the Methods. The number of migrated cells from three chambers in three independent experiments is shown. (A) Angiotensin II $\left(100 \mathrm{nmoll}^{-1}, 60 \mathrm{~min}\right)$ significantly stimulated cell migration to 1.4-folds greater than the control. This increase was blocked by coincubation with losartan $\left(100 \mathrm{nmoll}^{-1}\right)$, but not by PD123319 $\left(1 \mu \mathrm{mol} \mathrm{I}^{-1}\right)$. In addition, angiotensin II-induced cell migration was inhibited in the presence of sirtinol $(20 \mu \mathrm{moll}-1,24 \mathrm{~h}) .{ }^{*}$ and ${ }^{* *}$ indicate $P<0.05 \mathrm{vs}$. cells treated with vehicle alone and cells treated with PD123319 alone, respectively. (B) The transfection of siRNA against SIRT2 blocked angiotensin II-induced migration. In contrast, the transfection of control siRNA had no obvious effects on angiotensin II-induced migration. * and ** indicate $P<0.05$ vs. cells treated with vehicle alone and cells transfected with control siRNA, respectively.

siRNA also prevented mechanical stretch-induced changes of microtubules in EA hy.926 cells (Figure 7). These data indicate the involvement of angiotensin II type 1 receptor and SIRT2 in mechanical stretch-induced microtubule reorganization in endothelial cells.

\section{Angiotensin II-induced tubulin deacetylation in aortic intima} in mice in vivo

C57BL/6J mice were loaded with saline or angiotensin II $\left(1.44 \mathrm{mg} \mathrm{kg}^{-1} \mathrm{day}^{-1}\right)$ for 4 weeks to investigate the roles of angiotensin II-induced deacetylation of tubulin in vivo. Frozen sections of the abdominal aorta were stained with anti-acetylated tubulin and anti- $\alpha$ tubulin antibodies (Figure 8). In the control mice, acetylated tubulin was detected in the intima of the aorta (Figure 8A, a-f). In contrast, acetylated tubulin was decreased in the intima of aorta in mice loaded with angiotensin II (Figure 8A, g-l). Quantification of fluorescence intensity of the images supported these observations (Figure $8 \mathrm{~A}$ graph). Frozen sections were stained with the antibodies against 

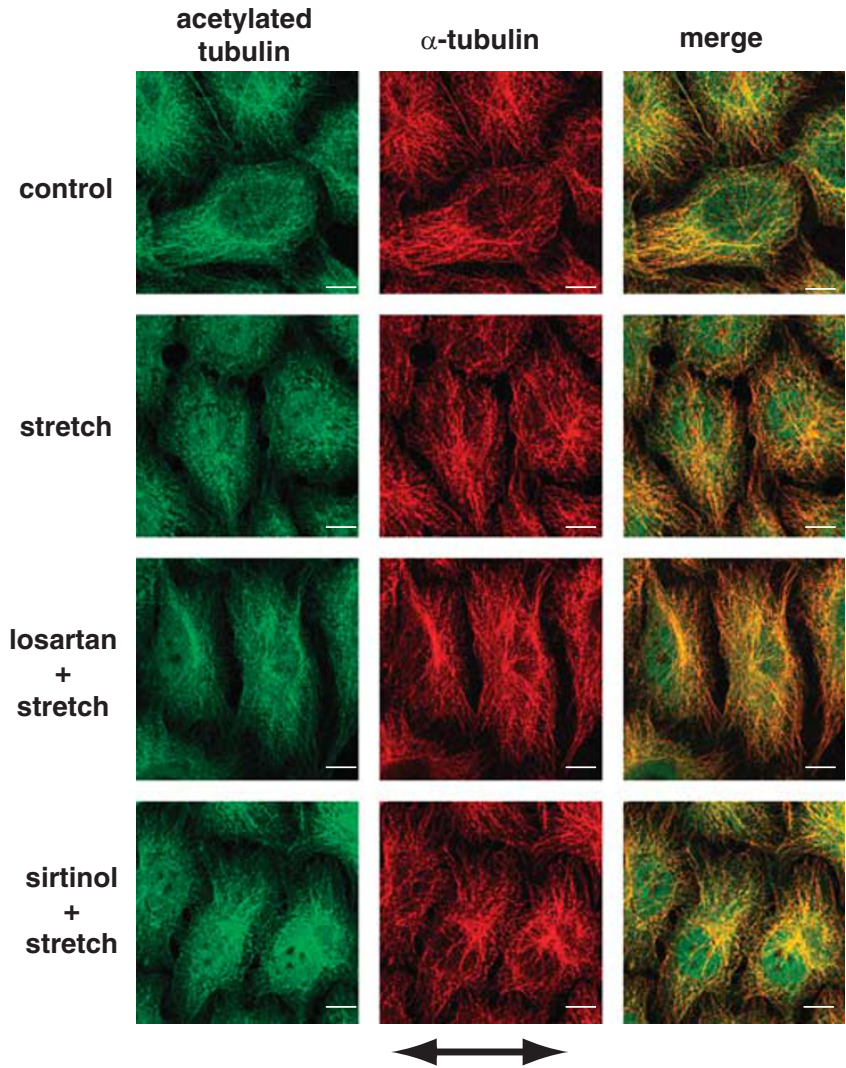

uniaxial cyclic stretch $; 10 \% \quad 0.83 \mathrm{~Hz}$

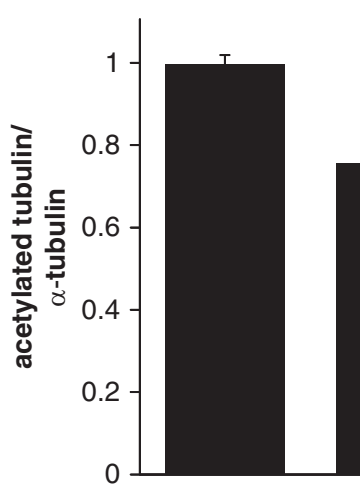

$\begin{array}{ccccc}\text { stretch } & - & + & + & + \\ \text { losartan } & - & - & + & - \\ \text { sirtinol } & - & - & - & +\end{array}$

Figure 6 EA hy. 926 cells pretreated with control vehicle, losartan or sirtinol were subjected to 0 or $10 \%$ uniaxial cyclic stretch for $2 \mathrm{~h}$ and then stained to detect acetylated tubulin (green) and $\alpha$-tubulin (red). Images observed by confocal microscopy are shown. Bar: $10 \mu \mathrm{m}$. Fluorescence intensity of acetylated tubulin adjusted with that of $\alpha$-tubulin measured as described in Methods was expressed relative to that of control (lower graph). ${ }^{*} P<0.05$ vs. non-stretched control cells.

acetylated tubulin and the endothelial marker, platelet-endothelial cell adhesion molecule-1 (PECAM-1), to clarify the cell types in aortic intima that were detected with anti-acetylated tubulin antibody (Figure 8B). Immunofluorescence confocal microscopy showed that acetylated tubulin was decreased in cells positively stained for PECAM-1 in the intima of the aortic walls in mice loaded with angiotensin II. These data suggest that angiotensin II stimulates the deacetylation of tubulin in aortic endothelial cells in mice in vivo.

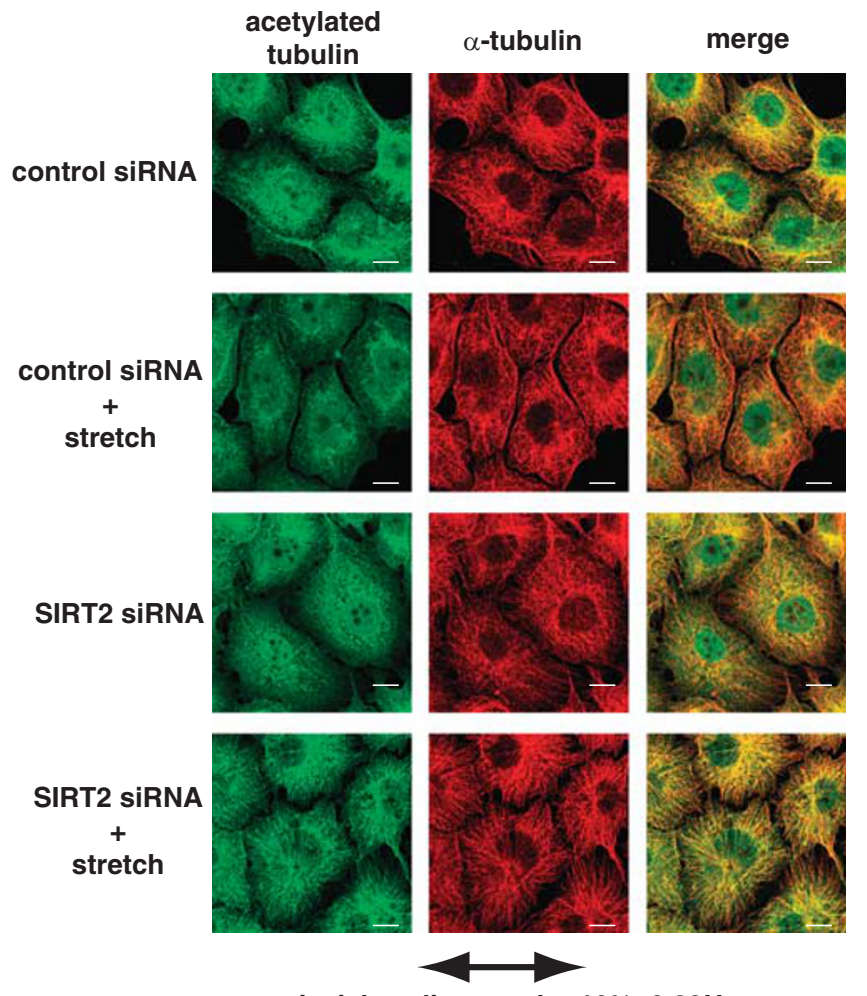

uniaxial cyclic stretch ; $10 \% \quad 0.83 \mathrm{~Hz}$

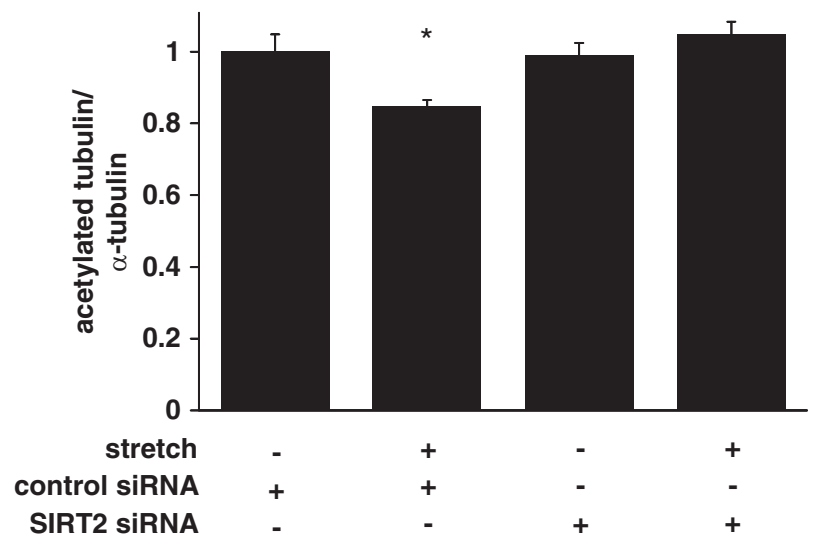

Figure 7 EA hy.926 cells transfected with control siRNA or siRNA against SIRT2 were subjected to 0 or $10 \%$ uniaxial cyclic stretch for $2 \mathrm{~h}$ and then stained with anti-acetylated tubulin (green) and anti- $\alpha$-tubulin (red) antibodies. Images observed by confocal microscopy are shown. Bar: $10 \mu \mathrm{m}$. Fluorescence intensity of acetylated tubulin adjusted with that of $\alpha$-tubulin measured as described in Methods was expressed relative to that of control (lower graph). ${ }^{*} P<0.05$ vs. non-stretched cells transfected with control SiRNA.

\section{DISCUSSION}

Vasoactive substances as well as mechanical stress triggered by hemodynamic forces such as mechanical stretch stimulate vascular cell signaling. Accordingly, chronic changes in vasoactive substances and hemodynamic forces caused by hypertension induce phenotypic changes in vascular cells that lead to vascular structural remodeling. Endothelial cells therefore have important roles in the maintenance of vascular structure and integrity. 
A

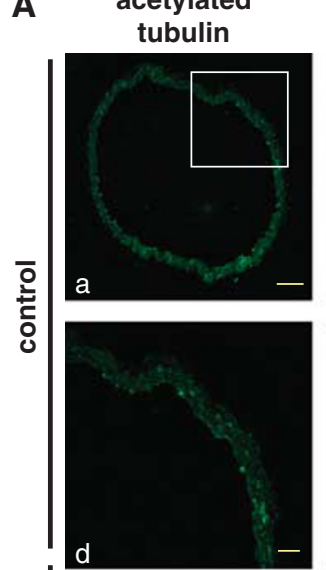

$\alpha$-tubulin
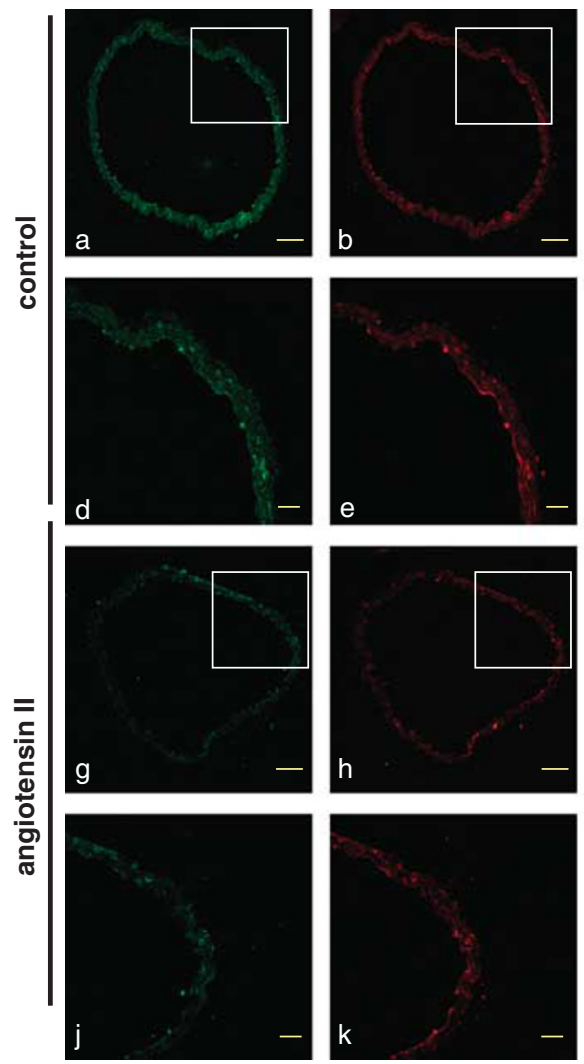

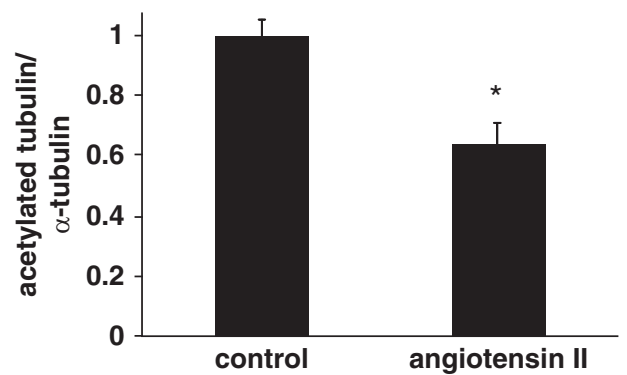

B
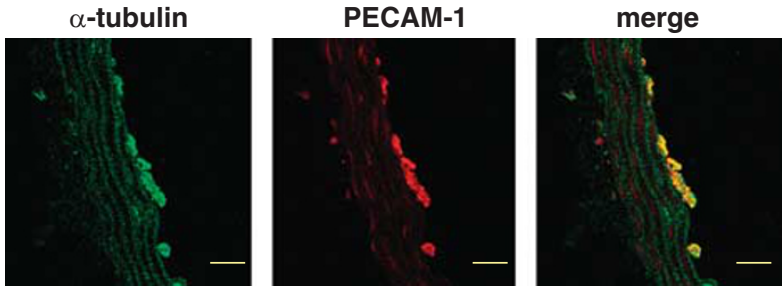

Figure $\mathbf{8}$ (A) Frozen sections of aortas prepared from mice loaded with control vehicle or angiotensin II were stained with anti-acetylated tubulin antibody (green) and anti- $\alpha$-tubulin antibody (red) and examined by confocal microscopy ( $n=3$ per group). Fluorescence intensity of images measured as described in Methods was expressed relative to that of control (lower graph). Acetylated tubulin was decreased in the aortic intima of mice loaded with angiotensin $\mathbf{I I}(\mathbf{g}-\mathbf{i} ; \mathbf{j}-\mathbf{I}$ depicted from the box in $\mathbf{g}-\mathbf{i}$, respectively) in comparison with control mice (a-c; $\mathbf{d}-\mathbf{f}$ depicted from the box in $\mathbf{a}-\mathbf{c}$, respectively). ${ }^{*} P<0.05$ vs. aorta loaded with control vehicle. (B) Frozen sections of the aortas were stained with anti- $\alpha$-tubulin (green) and antiPECAM-1 (red) antibodies and then were examined by confocal microscopy.
Microtubules, one of the key cytoskeletal fibers in endothelial cells, regulate numerous cellular functions, including cell shape, polarity, motility and intracellular vesicle transport. Much attention has been paid to the importance of posttranslational modification of tubulin in the regulation of microtubule-based cellular function. In quiescent cells, microtubules contain acetylated $\alpha$-tubulin. In contrast, tubulin is deacetylated in the leading edge of migrating fibroblasts and neuronal growth cones. ${ }^{13,14}$ Hyperacetylated microtubules decreased microtubule dynamics, increased microtubule stability and decreased cell migration. ${ }^{15}$ Therefore, tubulin acetylation/deacetylation is implicated in the control of microtubule function and, therefore, the regulatory mechanism of tubulin acetylation/deacetylation by acetylase/deacetylase has attracted much attention.

Angiotensin II not only in blood circulation but also locally produced in vascular walls activates multiple signaling pathways through type 1 and type 2 plasma membrane receptors, both of which are expressed in endothelial cells. This study showed that angiotensin II decreased acetylated tubulin in endothelial cells both in vitro and in vivo. mRNA expression of angiotensin II type 1 and type 2 receptors was not changed by SIRT2 silencing in EA hy.926 cells (data not shown). Pharmacological inhibition revealed that angiotensin II-induced microtubule reorganization and tubulin deacetylation are mediated by angiotensin II type 1 receptors. SIRT2 and histone deacetylase 6 (HDAC6) have been identified as tubulin deacetylases. Although caloric restriction has been shown to activate SIRT2 in a nicotinamide adenine dinucleotide ${ }^{+}$-dependent manner, the regulatory mechanism for SIRT2 deacetylase activity is not fully understood. ${ }^{16}$ This study showed that angiotensin II-angiotensin II type 1 receptor-induced tubulin deacetylation is mediated by SIRT2, suggesting that angiotensin II type 1 receptor signaling is a novel SIRT2 deacetylase regulator. In this study, SIRT2 expression was not changed by angiotensin II in EA hy.926 cells (data not shown). There is a possibility that angiotensin II signaling regulates SIRT2 activity through posttranslational modification of SIRT2 including phosphorylation. ${ }^{17}$ On the other hand, HDAC6, which was originally characterized as a histone deacetylase, is associated with tubulin in the cytoplasm and deacetylates tubulin. ${ }^{18}$ Although HDAC6 has been implicated in the regulation of cell migration, cell-cell interactions and gene transcription, HDAC6 appears to function not only as a deacetylase but also as a deacetylase activity-independent molecular scaffold in a cell type-specific manner. ${ }^{19}$ HDAC6 depletion of EA hy. 926 cells using siRNA showed no obvious effects on the angiotensin II-induced tubulin decatylation, suggesting that HDAC6 has a minor role in angiotensin II-induced microtubule reorganization in endothelial cells (data not shown). Endothelial migration has a role in the wound repair of injured vessels and the development of vascular remodeling. The roles of angiotensin II-angiotensin II type 1 receptor-SIRT2 axis in endothelial migration were investigated using pharmacological and genetic methods and the results showed that SIRT2 inhibition prevented angiotensin II-induced endothelial migration. These data suggest that SIRT2-mediated microtubule reorganization is implicated in the endothelial cell function.

Endothelial cells are exposed to mechanical forces such as cyclic stretch and shear stress. Excessive strain is involved in the pathogenesis of vascular remodeling and atherosclerosis. Previous reports have shown that mechanical stretch increases reactive oxygen species, induces reorganization of integrins and regulates cytoskeletal reorganization in endothelial cells. ${ }^{20-22}$ On the other hand, mechanical forces such as cyclic stretch and shear stress modulate the vascular reninangiotensin system. Delli Gatti et al. ${ }^{2}$ reported that cyclic stretch induces the release of angiotensin II in human endothelial cells. 
Therefore, cyclic stretch is considered to synergistically augment the effects of angiotensin II on vascular cells. In addition, the common signaling pathways triggered by both angiotensin II and cyclic stretch have also been suggested to have important roles in hypertensioninduced vascular remodeling. This study indicates that SIRT2 mediates microtubule reorganization induced by angiotensin II and cyclic stretch in endothelial cells, suggesting that SIRT2 is a key regulator of endothelial remodeling. Further studies are needed to elucidate the role of angiotensin II-induced deacetylation of tubulin and the involvement of SIRT2 in vascular remodeling by in vivo studies that investigate the effects of activation and inhibition of SIRT2 in vascular remodeling.

\section{CONFLICT OF INTEREST}

The authors declare no conflict of interest.

1 Heeneman S, Sluimer JC, Daemen AP. Angiotensin-converting enzyme and vascular remodeling. Circ Res 2007; 101: 441-454.

2 Delli Gatti C, Osto E, Kouroedov A, Eto M, Shaw S, Volpe M, Lûscher TF, Cosentino F. Pulsatile stretch induces release of angiotensin II and oxidative stress in human endothelial cells: effects of ACE inhibitor and AT1 receptor antagonism. Clin Exp Hypertens 2008; 30: 616-627.

3 Wong MK, Gotlieb Al. The reorganization of microfilaments, centrosomes, and microtubules during in vitro small wound reendothelialization. J Cell Biol 1988; 107: 1777-1783.

4 Mitchison T, Kirschner M. Dynamic instability of microtubule growth. Nature 1984; 312: 237-242.

5 Mitchison T, Kirschner M. Direct observation of steady-state microtubule dynamics. J Cell Biol 1986; 102: 1007-1019.

6 Horio T, Hotani H. Visualization of dynamic instability of individual microtubules by dark-field microscopy. Nature 1986; 321: 605-607.

7 Hommand JW, Cai D, Verhey KJ. Tubulin modifications and their cellular functions. Curr Opin Cell Biol 2008; 20: 71-76.

8 Verhey KJ, Gaetig J. The tubulin code. Cell Cycle 2007; 6: 2152-2160.
9 Inoue T, Hiratsuka M, Osaki M, Oshimura M. The molecular biology of mammalian SIRT proteins. Cell Cycle 2007; 6: 1011-1018.

10 North BJ, Marshall BL, Borra MT, Denu JM, Verdin E. The human Sirt2 ortholog, SIRT2, is an $\mathrm{NAD}^{+}$-dependent tubulin deacetylase. Mol Cell 2003; 11: 437-444.

11 Edgell CJS, McDonald CC, Graham JB. Permanent cell line expressing human factor VIII-related antigen established by hybridization. Proc Natl Acad Sci USA 1983; 80: 3734-3737.

12 Hirase T, Kawashima S, Wong E, Ueyama T, Rikitake Y, Tsukita S, Yokoyama M, Staddon J. Regulation of tight junction permeability and occludin phosphorylation by RhoA-p160ROCK dependent and independent mechanisms. J Biol Chem 2001; 276: 10423-10431.

13 Piperno G, Dizet M, Chang X. Microtubule containing acetylated $\alpha$-tubulin in mammalian cells in culture. J Cell Biol 1987; 104: 289-302.

14 Lim SS, Sammak PJ, Borisy GG. Progressive and spatially differentiated stability of microtubules in developing neuronal cells. J Cell Biol 1989; 109: 253-263.

15 Tran AD, Marmo TP, Salam AA, Che S, Finkelstein E, Kabarriti R, Xenias HS, Mazitschek R, Hubbert C, Kawaguchi Y, Sheetz MP, Yao TP, Bulinski C. HDAC6 deacetylation of tubulin modulates dynamics of cellular adhesions. J Cell Sci 2007; 120: 1469-1479.

16 Vaquero A, Scher MB, Lee DH, Sutton A, Cheng HL, Alt FW, Serrano L, Sternglanz R, Reinberg D. Sirt2 is a histone deacetylase with preference for histone $\mathrm{H} 4$ Lys 16 during mitosis. Genes Dev 2006; 20: 1256-1261.

17 Pandithage R, Lilischkis R, Harting $K$, Wolf $A$, Jedamzik $B$, Lüscher-Firzlaff J, Vervoorts J, Lasonder E, Kremmer E, Knöll B, Lüscher B. The regulation of SIRT2 function by cyclin-dependent kinases affects cell motility. J Cell Biol 2008; 180: 915-929.

18 Hubbert C, Guardiola A, Shao R, Kawaguchi Y, Ito A, Nixon A, Yoshida M, Wang XF, Yao TP. HDAC6 is a microtubule-associated deacetylase. Nature 2002; 417: 455-458.

19 Valenzuera-Fernandez A, Cabrero JR, Serrador JM, Sánchez-Madrid F. HDAC6: a key regulator of cytoskeleton, cell migration and cell-cell interactions. Trends Cell Biol 2008; 18: 291-297.

20 Cheng JJ, Wung BS, Chao YJ, Wang DL. Cyclic strain-induced reactive oxygen species involved in ICAM-1 gene induction in endothelial cells. Hypertension 1998; 31: 125-130.

21 Yano Y, Geibel J, Sumpio BE. Cyclic strain induces reorganization of integrin $\alpha 5 \beta 1$ and $\alpha 2 \beta 1$ in human umbilical vein endothelial cells. J Cell Biochem 1997; 64: 505-513.

22 Dartsch PC, Betz E. Response of cultured endothelial cells to mechanical stimulation. Basic Res Cardiol 1989; 84: 268-281. 